\title{
LA CONVERSATION ENTRE MARIE ET JÉSUS AUX NOCES DE CANA : ÉTUDE PRAGMATIQUE D'UN ENCHAÎNEMENT CONVERSATIONNEL SÉMANTIQUEMENT INCOHERENT
}

\author{
Jean-Marie Andoh GBAKRE \\ Université Peleforo Gon Coulibaly, Côte d'Ivoire \\ andoh225@yahoo.fr \\ Article reçu le 16 novembre 2016 | révisé depuis le 18 novembre 2016 | accepté le 24 décembre 2016 \\ RÉSUMÉ. La sémantique consacre l'explicite dans l'usage syntaxique des mots. \\ Cependant, il existe des niveaux d'actes de langage où la fluidité du lien sémantique \\ observe une rupture. La conversation entre Marie et Jésus, lors des noces de Cana, fait cas \\ d'étude. Face à la limite de l'interprétation sémantique constatée dans cet échange qui à \\ l'air d'un dialogue de sourd, et qui pourtant comporte une finalité perlocutoire \\ prégnante, la pragmatique est convoquée. Comment se perçoit la limite de \\ l'interprétation sémantique en conversation de façon générale, et, qu'est-ce qui caractérise \\ l'échange entre Marie et Jésus? Comment se fait dans ce dialogue le passage de \\ l'illocutoire au perlocutoire ? Le traitement de ces questions constitue le schéma \\ procédural de la réflexion qui entend dégager la solidarité de la pragmatique face aux
} limites de la sémantique.

Mots-clés: cognition, cohérence sémantique, efficacité pragmatique, interaction, variables actionnelles.

\begin{abstract}
The semantics devotes explicit in the syntactic use of words. However, there are levels of speech acts where the fluidity of the semantic link observes a break. The conversation between Mary and Jesus at the wedding at Cana is a case study. Faced with the limits of the semantic interpretation found in this exchange that looks a dialogue of the deaf, and yet has a purpose perlocutory pregnant, pragmatics is called. How to perceive the limits of semantic interpretation in general conversation and what characterizes the exchange between Mary and Jesus? How is this dialogue in the passage of the illocutionary perlocutory? The treatment of these issues is the procedural pattern of thinking which intends to generate the solidarity of pragmatic face to the limits of semantics.
\end{abstract}

Keywords: actionals variables, cognition, consistency semantics, interaction, pragmatic efficiency. 


\section{INTRODUCTION}

L'enjeu primordial d'une conversation est pour le locuteur de parvenir à se faire comprendre de son interlocuteur. Ce n'est qu'après cette première étape que découle la question des effets illocutionnaires soumis à un éventuel succès ou à un échec perlocutoire. En tout état de cause, l'énonciation met en jeu deux pôles dont le second est censé coopérer avec le premier afin de favoriser à celui-ci les chances d'un contrat de compétence à performance. De même, dans la recherche de cet équilibre conversationnel, il revient surtout au locuteur de proposer à l'interlocuteur un schéma conventionnel de description du sens.

A première vue, la caractéristique syntaxico-sémantique d'une phrase selon les perspectives saussuriennes (1916) et chomskyennes (1970) ne s'écarte pas de la logique du conversationnisme développée par Grice (1979) en référence aux maximes de quantité et de qualité issues du principe de signification naturelle. Mais dans l'interactivité pragmatique des choses aux mots, il arrive que le locuteur viole la loi de l'informativité et applique aux énoncés une hiérarchie de sens en arrière-plan conversationnel. L'enjeu qui émaille ce niveau interactionnel dépasse le principe formel de coopération sémantique pour emprunter une valeur de signification nonnaturelle, voire particulière. Ce niveau d'approche des faits de langue systématise une distanciation face aux données élémentaires et canoniques du sens de la phrase prescrites par Saussure et Chomsky. Dans ce procédé, l'implication forte d'un savoir épistémique copartagé par les interactants semble prévaloir sur le principe d'alternance. La conversation indique un certain balbutiement en surface symétrique, et pourtant, l'équilibre illocutoire des interventions assure au dialogue une dynamique cohésive souterraine. A vrai dire, le problème ne concerne pas la finalité perlocutoire qui régit les enchaînements, il se situe au niveau du mécanisme opératoire qui enclenche le jeu de l'idée à travers le mot. Et puisque c'est l'intention qui mobilise l'acte de parole, alors l'intention devient le porteur de l'enjeu du dit. Qu'une signification soit naturelle ou qu'elle soit non naturelle, l'enjeu perlocutoire entre les interactants reste le point focal du dialogal. Ainsi, c'est cette dualité complémentaire dans laquelle la linguistique pragmatique $s^{\prime}$ est investie en posture générale de théorie descriptive des faits de langue qui instruit cette réflexion à travers le faire biblique des noces de Cana.

Des questions qui se posent: à quel niveau d'interprétation la sémantique montre-t-elle un handicap à élucider certains énoncés en interaction ? Comment ce niveau $\mathrm{du}$ sens induit ou impliqué peut-il être appréhendé dans une conversation telle que celle qui a eu lieu entre Marie et Jésus aux noces de Cana? En quoi le principe de coopération prime-t-il sur le principe d'alternance? La détermination de l'enjeu perlocutoire des variables actionnelles entre Marie et Jésus provient de la saisie de l'illocutoire en tant qu'une dimension pertinente en conversation. Ces aboutissants de la réflexion procèdent de l'indication de la pragmatique comme une possibilité d'extension des bornes sémantiques en conversation.

La sémantique mobilise dans l'activité conversationnelle une logique opératoire qui détermine un sens conventionnel aux interventions. Les mots utilisés dans l'interaction ne sont pas dominés par la situation qui suscite leur emploi. L'interlocuteur est investi du même axe syntagmatique que le locuteur dans le choix que le second opère sur l'axe paradigmatique. Ce qui importe dans l'axiome sémantique, c'est la fluidité du mécanisme de saisi du message par B par rapport à une éventuelle désambigüité des énoncés mis en scène par $A$. C'est ce principe du dialogue emprunt de clarté qui a amené Cristea (2003) à proposer des clauses conversationnelles. Pour elle, une conversation doit avoir « des contraintes d'enchaînement » qui se définissent sous quatre aspects :

1) des contraintes thématiques: il doit y avoir une unité de thème entre le constituant initiatif et le constituant réactif:

-Quand est-il arrivé ?

- Hier au soir. 
-* J'ai la migraine.

2) des contraintes de contenu propositionnel: le constituant réactif doit être en relation sémantique avec le constituant initiatif:

- Quand est-il arrivé ?

- Hier au soir.

- * Après-demain.

3) des contraintes illocutoires: le constituant réactif doit avoir la même force illocutoire que le constituant initiatif.

-Tu devrais promettre de lui téléphoner. -Je le ferai ce soir même.

- * Je lui demanderai ce service

4) des contraintes d'orientation argumentative: le constituant réactif doit être co-orienté au constituant initiatif, en ce sens qu'on ne peut défendre deux conclusions opposées à l'aide du même argument ou inversement utiliser deux arguments opposés pour servir la même conclusion :

- Il y a du verglas et j'ai peur d'aller trop vite.

- Oui, il faut être prudent.

- Donc je vais ralentir.

- * Donc je vais accélérer.

- * Si l'on veut être prudent, quand il y a

du verglas il faut rouler à vitesse réduite et accélérer.

(p.146)

Toutefois, dans la pratique, ces prescriptions ne sont pas toujours respectées et donnent lieu à des interprétations situationnelles prégnantes. Dans les échanges suivants, des ambigüités de sens amènent à se référer au contexte de la conversation.

A : La pluie s'annonce alors qu'il est l'heure de partir.

B : Malheureusement, Koffi est sorti avec le parapluie.

$\mathrm{A}^{\prime}$ : La pluie s'annonce alors qu'il est $l^{\prime}$ heure de partir.

$B^{\prime}$ : Oui. Surtout que Koffi nous a devancés.

L'approche sémantique telle que définie dans la linguistique saussurienne (1916) se caractérise par le principe de compositionnalité du sens et le principe de littéralité. L'éventualité du départ révélé en A est obstrué par l'événement «pluie » et le défaut de l'élément " parapluie » indiqué par B. B est une séquence imputative de la cause émise en A. Dans la démarche de sens, A administre un postulat dont B est une réponse explicite du besoin évoqué ainsi que de la raison signifiée «Koffi est sorti avec le parapluie». Mais, au lieu du relais énonciatif $\mathrm{B}$, si $\mathrm{A}$ est face à une réponse dans l'ordre de $B^{\prime}$, alors il faut recourir à la thèse de Ducrot (1984, p.176) selon laquelle «l'idée d'une composante sémantique autonome: le niveau sémantique, et dans certains cas syntaxique, doit contenir des indications proprement pragmatiques ». Dans cette même perspective, Ducrot prétend que "donner la signification lexicale des mots n'est pas donner le sens réel de ceux-ci mais mettre à jour les instructions qui sont nécessaires pour calculer le sens des énoncés en situation". La situation en B' est que "Koffi », actant non présent mais dont l'absence influence l'instance conversationnelle, empêche $\mathrm{A}^{\prime}$ et $B^{\prime} d^{\prime}$ accomplir l'action de sortir. La notion $\mathrm{d}^{\prime}$ « indications proprement pragmatiques » évoquée par Ducrot est de toute notoriété, ce dans la mesure où le "proto énoncé" B', c'està-dire la charge de signification induite, se situe non pas au niveau sémantique, mais dans la tension illocutoire exercée parles interactants $\mathrm{A}^{\prime} / \mathrm{B}^{\prime}$. En effet, dans l'entendement de $B^{\prime}, A^{\prime}$ est supposé savoir qu'il existe un parapluie. Par ailleurs, énoncer «Koffi nous a devancés» et soutenir cet état de fait comme raison de l'impossibilité de se rendre mobile sous la pluie autorise la reconstruction énonciative explicite :

$\mathrm{B}^{\prime \prime}$ : Oui. Surtout que Koffi nous a devancés avec le parapluie.

Pour que l'intervention de B' présente une aisance dans l'interprétation, il aurait fallu indiquer clairement dans l'acte subordonné qu'il existait un parapluie, comme il est le cas en $\mathrm{B}^{\prime \prime}$, mais que cet élément qui aurait pu permettre une sortie aisée sous la pluie a été déjà utilisé par l'actant absent «Koffi ». Cependant, s'arrêter à une réplique dans le sens immédiat de: «Oui. Surtout que Koffi nous a devancés », ne détermine pas explicitement en quoi "Koffi » incarne une difficulté pour les interacteurs présents. Les deux actants présents sont instruits d'une dimension cognitive qui facilite l'échange, mais, la substance significative qui ressort de leur 
dialogue n'est pas ipso facto portée à la mesure de compréhension d'un sujet passif au dialogue.

Dans un autre cas de figure, les échanges peuvent comporter un posé sémantique perçu, sans pour autant que la réaction de l'interlocuteur dévoile toute la charge du dialogue. Dans la suite,

$\mathrm{A}^{1}$ : A mon retour de la banque, tu iras au supermarché.

$\mathrm{B}^{1}$ : C'est compris, je t'attends.

il y a entre les interactants une complicité situationnelle qui affecte au dialogue un sens partiel. En effet, $\mathrm{A}^{1}$ indique une condition qui sous tend deux enjeux. Le premier, explicite, est son retour de la banque. Le second, implicite, est le succès de l'opération bancaire que celui-ci va entreprendre. $\mathrm{Si} \mathrm{B}^{1}$ doit attendre que $\mathrm{A}^{1}$ revienne de la banque avant de se rendre au supermarché, c'est non seulement parce que l'environnement immédiat où les échanges se déroulent comporte des orientations à satisfaire, mais c'est surtout parce que la banque est le nœud de réalisation de l'action que doit mener $B^{1}$. La réponse de $\mathrm{B}^{1}$ est donc une consécution relative qui porte un enjeu cognitif. Elle propose explicitement une information admise au sens commun, mais elle implique une condition de satisfaction instituée par l'acte directeur de l'échange. Ainsi, "la sémantique doit considérer, en plus du locuteur, des auditeur(s), du moment et du lieu de chaque contexte possible d'énonciation, son arrière-plan conversationnel (les énonciations antérieures, les formes de vie et les attitudes partagées par les interlocuteurs, notamment leurs connaissances mutuelles)» (Vanderveken, 2007, p.241).

Dans ces conversations, les locuteurs et leurs interlocuteurs partagent les mêmes réalités de circonstance, laquelle circonstance conditionne le processus de signification des interventions. Toute intervention est le relais d'une symétrie cognitive qui instruit un niveau d'échanges non astreint à l'orthodoxie syntaxico-sémantique.

« L'ordre conversationnel est le produit des actions méthodiques des co-participants : est en jeu une dimension interprétative et donc cognitive» (De Fornel et Léon, 2000, p.153).
L'enjeu est donc de cerner la visée illocutoire consécutive à une modalité expressive appropriée. Et c'est cette dimension de la réflexion qui instruit la suite du travail à l'analyse des noces de Cana.

\section{MÉTHODE}

La pragmatique, méthode princeps qui fonde cette étude, est une dimension enrichie de la linguistique dans le sens où, en rapport aux sentiers battus des formes phraséologiques plates dans lesquelles s'est confiné le structuralisme, elle examine le mécanisme d'agencement des mots qui conduit à la fois au sens perçu et au sens induit. Plusieurs écoles ont émergé et chacune d'elles s'est approprié un pan spécifique de son rayonnement. Ce travail s'inscrit dans le registre de l'analyse conversationnelle conformément aux règles d'échanges prescrites par l'école de Genève. Avec sa revue Cahiers de Linguistique française, cette école constitue un véritable lieu de consécration des recherches en pragmatique analytique. En additif à ce vivier d'expérimentation du langage en situation, des travaux de linguistes, à l'instar de Ducrot (1984), Kerbart-Orecchioni (2001), etc. permettent une fluidité transversale dans la détermination des jeux de sens.

C'est le lieu de rappeler que le prétexte de toute étude en pragmatique est féconde non du posé, mais de l'instrumentalisation qui permet la mise en sens de l'objet perçu. Quand un discours est produit, il se range automatiquement au lot d'antériorité, ce qui suppose la susceptibilité qu'il soit perçu comme un discours rapporté, peu importe la justification factuelle que l'on voudrait lui porter. Mais ce qui importe, et qui certainement a inspiré cette réflexion, $c^{\prime}$ est le caractère événementiel qui instruit la vivacité illocutoire de l'interaction entre Marie et Jésus. Selon Benveniste (1969), « elle [la langue] se manifeste par l'énonciation qui porte référence à une situation donnée ; parler c'est toujours parler de" (p.62). Combien serait-il alors délicat d'étudier l'énonciation ou encore le discours sans se servir de l'énoncé? Le produit est ostensiblement un chemin de la substance visée. En cela, la dimension inattendue des 
noces de Cana est porteuse d'analyse en pragmatique conversationnelle. Au niveau discursif, les enchaînements souffrent d'une incohérence prescriptive, laquelle incohérence n'influence pas pour autant le résultat de l'action verbale de Marie. Cette force de l'enjeu complice au jeu est décryptée en confrontation aux principes de l'informativité, de l'alternance, de la coopération et des visées illocutoires et perlocutoires.

\section{RÉSULTATS ET DISCUSSION Le principe d'alternance face à la force illocutoire}

La conversation entre Marie et Jésus, aux noces de Cana, relève d'une implicature conversationnelle particulière. La logique d'enchaînement n'est pas respectée. Mais, cela n'empêche pas les actants de mener la conversation et d'induire une portée actionnelle.

(Marie) -Ils n'ont pas de vin.
(Jésus) -Qu'ya-t- il entre moi et toi,
femme? Mon heure n'est pas encore
venue.

(Marie) -Faites tout ce qu'il vous dira.

(Jean 2 : 3-5, Les éditions du Cerf)

Si nous considérons l'acte d'intervention «Qu'y a-t-il entre moi et toi, femme? », il y a matière à dire que Jésus, pris dans l'élan des festivités se voit indisposé par sa mère qui l'interpelle. En effet, le principe de l'enchaînement aurait été sémantiquement correct si la réplique faite proposait un schéma explicite dans l'ordre marqué de la saisie de l'information. Mais la spontanéité apparemment réfutative qui caractérise sa réponse semble se soustraire aux normes de l'enchaînement conversationnel. Il y a une sorte de rupture dans la progression sémantique naturelle du dialogue.

Cependant, en faisant progresser ce raisonnement, il apparaît une crise de sens, puisque Jésus va à la suite des échanges exercer sur lui un dépassement pour donner une ouverture de faisabilité à la requête implicite induite : "Mon heure n'est pas encore venue». Après avoir marqué un fossé sémantique dans l'enchaînement du dialogue, il offre une possibilité de satisfaction de l'acte initiatif. Et c'est dans ce second segment de son intervention que se situe la logique illocutoire qui instruit l'acte directeur de l'échange instauré par Marie.

En effet, la dimension factuelle de l'intervention de Marie est forte d'une consécution sous jacente. Le Christ n'est pas le maître du repas. Aussi sa présence aux noces de Cana est-elle logée à la même enseigne que celle des invités lambda. Cependant, la relation particulière entre Marie et lui permet à cette dernière de violer les principes de civilités pour militer à la cause d'une urgence situationnelle. L'annonce de la rupture du vin, le vin, source de convivialité indéniable en pareille circonstance, va autoriser une délégation de pouvoir et une révélation de pouvoir. «Bien entendu, les unités premières réelles de la conversation sont les actes illocutoires qui sont tentés plutôt que ceux qui sont exprimés. La signification du locuteur est souvent différente de celle de l'énoncé utilisé» (Vanderveken, 2007, p.231). C'est donc à la sollicitation de l'exercice de pouvoir divin dont Marie est instruite que Jésus répond.

Par ailleurs, la relation de succession interactive entre ces deux acteurs émane d'un lien empirique. Il n'y a que le courant illocutoire qui traverse cet échange qui fonde de raison la méthode fournie et le résultat de l'acte initiatif. La séquentialité de la conversation est le produit d'enchaînements abscons. Les interventions sont marquées par une charge illocutoire dont la distribution sémantique, en termes de satisfaction des données initiales, est intimement liée aux savoirs de croyance des intervenants. Cela dit, l'adresse de Marie aux serviteurs: «Faites tout ce qu'il vous dira» est une prémonition au perlocutoire qui instruit un équilibre épistémique partagé avec ceux-ci. De fait, ces derniers sont présents depuis le début de la scène. Ce qui suppose que l'acte subordonné de Jésus, «mon heure n'est pas encore venue» a été prononcé en leur présence. Mais, ils préfèrent opter pour l'enchaînement que fait Marie, malgré que Jésus ne donne pas à travers son intervention l'évidence d'un contrat interactif réussi.

Qui plus est, la requête elle-même est donnée sous la forme d'une information. En 
effet, Marie aurait pu dire: «Peux-tu leur procurer du vin?", ce qui supposerait que Jésus possède le vin en tant que matière physique, et est susceptible de l'offrir à l'assemblée. Mais ce n'est pas le cas. Elle procède plutôt par une stratégie interactive dans laquelle l'acte directeur «ils n'ont pas de vin», en réalité, est infléchi à l'acte subordonné «Mon heure n'est pas encore venue». De fait, au lieu de l'assertionrequête : "ils n'ont pas de vin », il aurait été plus plausible de procéder par une prérequête en termes de prémisses conversationnelles: "Ton heure n'est-elle pas encore venue?». Cela aurait permis une conduite sémantique mieux affinée de l'acte réactif «Mon heure n'est pas encore venue ». Ainsi fait-elle le choix d'entretenir le dialogue d'une tension pragmatique qui, toutefois, reste sous le contrôle épistémique des différents protagonistes. En bref, c'est la réaction de Jésus «Mon heure n'est pas encore venue » qui montre qu'il a interprété «Ils n'ont pas de vin » comme une requête. L'acte directeur que Marie engage comporte, selon l'expression de Vanderveken (2007, p. 236), « une force illocutoire $F$ à travers un contenu propositionnel $\mathrm{P}, \mathrm{F}(\mathrm{P}) »$. Pour Vanderveken (2007) :

Un locuteur accomplit avec succès un acte illocutoire de la forme $F(P)$ dont $F$ représente la force et $P$ le contenu propositionnel en un contexte donné d'énonciation quand, en ce contexte, premièrement, le locuteur réussit à atteindre le but illocutoire de cette force sur le contenu avec le mode d'atteinte qui lui est propre, deuxièmement, la proposition $P$ satisfait les conditions sur le contenu propositionnel de $F$, troisièmement, le locuteur présuppose alors toutes les propositions déterminées par les conditions préparatoires de cette force relativement au contenu et, finalement, il exprime également avec le degré de puissance requis par $F$ toutes les attitudes déterminées par les conditions de sincérité de cette force à propos de $P$. (p.236)

Marie a une adresse détournée à l'endroit de Jésus. Elle énonce "un acte de langage a priori assertif »(Brassac, 1992, p.69). Jésus est certes son fils, mais elle est investie du pouvoir culturel de la tradition juive qui demande à la femme de la discrétion et de l'égard dans les rapports interactifs, surtout face à $l^{\prime}$ Homme. Aussi semble-t-elle prise à un dilemme. Dans le même moment qu'elle voudrait collaborer à la pérennité de l'atmosphère de joie qui règne, elle ne voudrait pas livrer la face divine de son fils au public. Et pourtant, la révélation de Jésus au monde est ici un passage obligatoire pour l'accomplissement illocutoire de la conversation.

$\mathrm{Au}$ demeurant, l'attitude conversationnelle qu'elle adopte, permet de retenir à la suite de Alferov et Kustova (2016, p.12) que «Communiquer, ce n'est pas seulement interagir avec des interlocuteurs, présents ou potentiels, c'est entreprendre avec eux une véritable négociation, et ceci à plusieurs niveaux (...) ». En effet, par rapport au défi de l'enjeu conversationnel, la requête faite met inéluctablement en jeu la face de Jésus. Si la demande est satisfaite, cela suppose que Jésus aurait entrepris de ménager sa face positive. Dans un autre cas de figure, un refus pourrait influer négativement sur son image. Le public des noces est informé de sa présence. Mieux, il est instruit des croyances émises à son actif, notamment sa dimension divine. Marie en a conscience, ce qui l'oblige à la prudence interactive à travers le choix de l'implicite conversationnelle. Par ailleurs, si elle engage une telle modalité énonciative, c'est parce

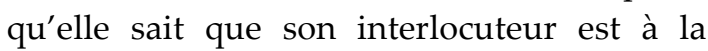
hauteur de l'implicature qu'elle y adapte.

La réaction de Jésus est ainsi naturellement inscrite à la mesure de l'acte initiatif: "Qu'y a-t- il entre moi et toi, femme? Mon heure n'est pas encore venue». Dans un premier temps, cette intervention indique une surprise du locuteur. Jésus est surpris que Marie sois instruite de sa divinité. Dans un second moment, Jésus se sent découvert, car en réalité, ce que Marie a énoncé, le manque de vin, lui également l'a constaté et intérieurement il s'en est probablement offusqué. Seulement, son acte réactif témoigne de ce que, ce qui était en lui caché, a été mis à nu. La dynamique qui résulte de ce passage peut se résumer à travers l'approche d'Alferov sur le déroulement d'un dialogue référentiel. Selon 
ce linguiste: «En premier lieu les interlocuteurs confrontent leurs attitudes propositionnelles sur le référent et par là essaient d'influer sur leurs croyances respectives. (...) (En second lieu) L'échange de paroles porte sur les modalités d'énonciation qui correspondent à la relation interpersonnelle entre les protagonistes de la communication » (Alferov et Kustova, p.2016, p.11). En somme, la conversation n'est pas que situationnelle. Elle est soumise à des affluents tels que le lien intersubjectif et les savoirs épistémiques que partagent les acteurs au dialogue. Limiter l'interprétation des données interactionnelles aux actes perçus pourrait occasionner une fuite de signification, puisque ici par exemple, entre Marie et Jésus, il existe une attraction psychologique qui imprime aux interventions une logique d'action naturelle, ce qui n'est pas forcément une logique naturelle pour le sens commun. En substance, la coopération montre un dynamisme plus valorisant dans le processus interactif $\mathrm{qu}^{\prime} \mathrm{au}$ niveau $\mathrm{du}$ construit ostentatoire.

\section{Du principe de coopération à l'enjeu perlocutoire}

La coopération en conversation est la recherche d'un équilibre transversal entre les sujets lors $\mathrm{du}$ traitement thématique de l'élément référentiel. Elle vise une issue généralement heureuse, puisque la notion même de "coopérer" entraîne une idée de consensus. Charaudeau et Maingueneau (2002) définit le principe de coopération comme la mise en fonction d'une «construction collective et "collaborative" des conversations »(p.370), c'est-à-dire qu'il engage une complicité d'action verbale entre les interlocuteurs. Par ailleurs, là où réside l'intérêt de l'analyse à ce niveau, c'est qu'il peut avoir coopération implicite et résultat probant, sans pour autant que le processus interactif présente une homogénéité rationnelle.

En effet, la scène des noces de Cana hérite d'un contexte présuppositionnel qui révèle $d$ 'une certaine manière le caractère divin des actants. L'enjeu perlocutoire de l'intrusion de Marie est d'amener Jésus à mettre à la disposition des invités du vin de manière inopinée. En cela, la réplique subordonnée «Qu'y a-t- il entre moi et toi, femme?» en dépit du caractère de convention non naturelle est indicative d'une implicitation conversationnelle particulière révélatrice. Comme il a été indiqué en amont, Jésus n'interroge pas Marie dans le sens de la désavouer. Son interrogation, premièrement, marque un étonnement à savoir que Marie a percé son mystère, deuxièmement, ayant perçu son intimité violée, il est contraint à agir pour sauver, et la situation, et sa face.

$C^{\prime}$ est donc le mystère qui instruit la coopération implicite entre les deux sujets qui est remarquable. En effet, quel rapport pourrait-il y avoir entre la rupture du «vin » et une certaine «heure» qui ne serait pas encore «venue»? Et pourtant, dans le déroulement du processus scénographique, il y a une satisfaction de l'assertion initiale qui instruit le dialogue. La suite du texte souligne :

Or il y avait là six jarres de pierre, (...). Jésus leur dit: «Remplissez d'eau ces jarres (...) Puisez maintenant et portezen au maître du repas ». (...) - Le maître du repas appelle le marié et lui dit : « Tout homme sert d'abord le bon vin et, quand les gens sont ivres, le moins bon. Toi, tu as gardé le bon vin jusqu'à présent!

(Jean 2 : 6-11, Les éditions du Cerf)

Cela dit, quand Marie instruit Jésus du manque de vin, elle instaure une ostension connotative bidimensionnelle. D'abord, elle se permet cette requête parce qu'elle sait son interlocuteur capable de la réaliser. Par conséquent, vu que le contenu de l'information est vrai, une première condition de succès du dialogue est immédiatement validée. Ensuite, par rapport à la réaction de Jésus, réaction qui porte l'enjeu d'un faire faire, Marie révèle ainsi la subordination de Jésus à son endroit. La suite illocutoire «Faites tout ce qu'il vous dira» est une authentification des faits.

En outre, dans cet acte de clôture, elle induit une anticipation dans l'exercice de réalisation de sa requête, conséquemment à l'assise encyclopédique qu'elle possède en son for intérieur de son fils. Dans le cotexte, il $n^{\prime} y$ a certes aucune trace explicite qui fonde 
la raison qui la motive à tenir un tel propos. Mais, même si Jésus semble ne pas opérer dans la même logique qu'elle, il finit par ajuster son comportement à l'acte initiatif. A ce sujet, les interventions de Marie peuvent être considérées comme des actes dans la mesure où: «ils sont faits pour agir sur autrui [Jésus], mais aussi l'amener à réagir : quand dire, c'est faire, mais aussi faire faire ; c'est-à-dire que la production d'un acte donné crée sur la suite un certain nombre de contraintes, et un système d'attentes (...)» (Kerbrat-Orecchioni, 2001, p.58).

Marie est animée d'une conviction forte qui donne de la pertinence à ses actes de parole. La conversation qu'elle initie est «l'accomplissement séquentiel d'une extension de la logique illocutoire ». Trognon et Brassac (1992, p.77). Elle parle peu, mais ses interventions ont une efficacité prégnante. Après sa première intervention, elle revient à la charge pour tenir aux serviteurs un discours d'autorité: «Faites tout ce qu'il vous dira ». Ici, la particularité des échanges est relative à la convocation d'une auto détermination à croire en l'effet perlocutoire d'un acte de langage prononcé. Dit autrement, dans ce passage biblique, la dimension illocutoire des séquences constitue des paires adjacentes qui défient la logique sémantique à travers une prééminence des «efforts de coopération », dans le sens de Grice (1979). Selon lui :

Nos échanges de paroles ne se réduisent pas en temps normal à une suite de remarques décousues, et ne seraient pas rationnels si tel était le cas. Ils sont le résultat, jusqu'à un certain point au moins, d'efforts de coopération. (p.61)

Marie et Jésus s'influencent mutuellement dans un jeu de rôle implicite. Engagés dans une tension illocutoire, ils exercent un rapport d'équilibre conversationnel dont la force se mesure au degré cognitif. Jésus tente d'échapper à la thématique que propose Marie sans pour autant se détacher de l'espace interpersonnel. Les mots sont avancés avec prudence car à chaque fois qu'un mot est prononcé il promène un contingent psychologique censé entraîner une action. En effet, les rôles et les positions sociaux psychologiques de ces deux interactants confessent une relation intersubjective qui entrevoie un lien pragmatique empirique dont la scène dialogale des noces de Cana se révèle être l'espace discursif d'expérimentation. Les interventions, que ce soit en acte initiatif comme en acte réactif, construisent une représentation mentale des interactants. La substance actionnelle drivée : «faites tout ce qu'il vous dira », n'est autre que le produit dérivé des connotations qui régissent l'espace discursif. Les actes de parole engagent les caractères des protagonistes et déterminent procéduralement l'enjeu de la conversation. Il s'agit $\mathrm{d}^{\prime}$ « un processus circulaire où chaque message, chaque comportement d'un protagoniste agit comme un stimulus sur son destinataire et appelle une réaction qui, à son tour, devient un stimulus pour le premier» (Alferov et Kustova, 2016, p.6).

Dans cette logique, il se dégage une dynamique illocutoire qui sous-tend le parallélisme des actes de langage. En surface, la logique d'alternance est défectueuse, mais en structure profonde, le principe de coopération est un succès. La preuve n'est nulle par ailleurs que dans la finalité perlocutoire, en termes d'acte de parole réactif qui donne à Jésus de mettre fin au suspens. "Jésus leur dit (aux serviteurs) : « remplissez d'eau ces jarres (...)». Durant tout le processus interactif les traces interprétatives, dans la conduite thématique du faire, sont restées inscrites au paradigme épistémique des interactants. Mais au final, c'est l'enjeu du processus qui a triomphé du mode d'interaction choisi par les acteurs du dialogue.

\section{CONCLUSION}

Malgré l'absurdité conversationnelle qui a administré le dialogue entre Marie et Jésus, un nouvel environnement cognitif est établi, un nouvel état de chose existe: "la transformation de l'eau en vin par Jésus sous l'impulsion de Marie". La réflexion faite sur les noces de Cana révèle au niveau pragmatique deux éléments essentiels. Premièrement, il est question de la non influence $\mathrm{du}$ principe d'alternance sur le principe de coopération. Deuxièmement, il s'agit de l'enjeu perlocutoire, en termes de 
résultats des interactions, qui reste un effet tangible du principe de coopération entre les acteurs du dialogue. Ces deux réponses ellesmêmes se classent dans un ensemble conceptuel plus généralisant qui est l'affranchissement de la logique sémantique dans une perspective d'intégration à la visée pragmatique des enchaînements conversationnels. La complexité de l'intervalle conversationnel requis par la satisfaction de l'acte initiatif a indiqué une valeur additive dans l'approche conversationnelle non limitée aux fondamentaux sémantiques en conversation. La prédominance de l'intuition initiative de Marie conséquemment à un degré de prémonition suscitée, s'est soldée par un équilibre cognitif co partagé, ce qui a occasionné un succès perlocutoire. La logique d'enchaînement en conversation ne gouverne pas la force des mots. Les sujets sont appelés à une collaboration épistémique qui appelle à un équilibre du substrat psychologique qui est mis en œuvre. L'action coopère avec la force illocutoire qui émaille les interventions. En un mot, toute irrégularité dans la coordination des interventions dans une conversation n'est pas synonyme d'irrationalité tant que les interlocuteurs parviennent à donner à leurs échanges une issue de félicité actionnelle assortie à la complicité situationnelle.

\section{REMERCIEMENTS}

Nos reconnaissances vont à l'endroit de 1'Université Peleforo Gon Coulibaly dans sa dynamique d'impulser la recherche scientifique à travers les moyens infrastructurels et financiers déployés. Spécifiquement, nous saluons Messieurs Irié Bi Gohy Mathias (Université Alassane Ouattara) et Bohui Djédjé Hilaire (Université Félix Houphouët Boigny), Professeurs titulaires en Grammaire et Linguistique, pour les précieux conseils prodigués à l'initiative d'améliorer la qualité de cet article.

\section{RÉFÉRENCES}

Alferov, A.V., et Kustova, E.Y. (2016). Principes interactionnistes de l'analyse conversationnelle. Repéré à https://www.academia.edu/4289099/P RINCIPES_INTERACTIONNISTES_D E_LANALYSE_CONVERSATIONNEL LE.

Benveniste, E. (1969). Sémiologie de la langue. Semiotica. Revue de l'Association internationale de sémiotique, 1,2. Repéré à https://www.deepdyve.com/lp/degruyter/s-miologie-de-la-langue-2bPBRJWlbgg.

Brassac, C. (1992). Analyse de conversations et théorie des actes de langage. Cahiers de linguistique française, 13,62-75. Repéré à http://clf.unige.ch/numeros/13/.

Charaudeau, P. et Maingueneau, D. (2002). Dictionnaire d'analyse du discours. Paris : Editions du Seuil.

Cristea, T. (2003). L'analyse conversationnelle. Dialogos, 8, 138-151. Repéré à www.romanice.ase.ro/dialogos/08/23

De Fornel, M. et Léon, J. (2000). L'analyse de conversation, de l'ethnométhodologie à la linguistique interactionnelle. Histoire Épistémologie Langage, 22(1), 131-155. doi : 10.3406/hel.2000.2770.

Ducrot, O. (1984). Le dire et le dit. Paris: Éditions de Minuit.

Grice, H.P. (1979). Logique et conversation. Communications, 30(1), 57-72. doi : 10.3406/comm.1979.1446

Kerbart-Orecchioni, C. (2001). Les actes de langage dans le discours, Théorie et fonctionnement. Paris : Nathan/VUEF.

Trognon, A. et Brassac, C. (1992). L'enchaînement conversationnel. Cahiers de linguistique française,13, 76106. Repéré à http://clf.unige.ch/numeros/13/

Vanderveken, D. (2007). Principes de pragmatique formelle $\mathrm{du}$ discours. Philosophiques, 34(2), 229-258. doi : 10.7202/015880ar 